VoL. 39 (1989) [59-70]

\title{
A DISTANCE FUNCTION PROPERTY IMPLYING DIFFERENTIABILITY
}

\author{
J.R. GILES
}

In a real normed linear space $X$, properties of a non-empty closed set $K$ are closely related to those of the distance function $d$ which it generates. If $X$ has a uniformly Gâteaux (uniformly Fréchet) differentiable norm, then $d$ is Gâteaux (Fréchet) differentiable at $x \in X \backslash K$ if there exists an $\vec{x} \in X,\|\vec{x}\|=1$ such that

$$
\lim _{t \rightarrow 0+} \frac{d(x+t \vec{x})-d(x)}{t}=1
$$

and is Gâteaux (Fréchet) differentiable on $X \backslash K$ if there exists a set $P_{+}(K)$ dense in $X \backslash K$ where such a limit is approached uniformly for all $x \in P_{+}(K)$. When $X$ is complete this last property implies that $K$ is convex.

Given a real normed linear space $X$ a non-empty closed subset $K$ generates a distance function $d$ on $X$ where

$$
d(x)=\inf \{\|x-y\|: y \in K\} .
$$

A distance function $d$ always satisfies the Lipschitz condition

$$
|d(x)-d(y)| \leqslant\|x-y\| \quad \text { for all } x, y \in X
$$

and is a convex function if and only if $K$ is a convex set.

There is a natural duality between the properties of the set $K$ and its distance function $d$. We see this duality exploited in abstract approximation theory and in techniques of non-smooth optimisation.

A longstanding abstract approximation problem concerns Chebyshev sets. A subset $K$ is said to be a Chebyshev set if for each $x \in X \backslash K$ there exists a unique $p(x) \in K$ such that

$$
d(x)=\|x-p(x)\| .
$$

The problem is to determine necessary and sufficient conditions for a Chebyshev set to be convex. The best result so far was given by Vlasov $[10,11]$ who showed that in

Received 23 March 1988

Copyright Clearance Centre, Inc. Serial-fee code: $0004-9729 / 89 \$ \$ 2.00+0.00$. 
a Banach space $X$ with rotund dual $X^{*}$ a Chebyshev set $K$ with continuous metric projection $x \rightarrow p(x)$ is convex. He used the continuity of the metric projection to derive a differentiability condition for the distance function $d$ and this differentiability condition for $d$ was actually the key to his proof for convexity of $K$.

As a tool for non-smooth optimisation Clarke [4] developed the generalised subdifferential for a real locally Lipschitz function $\phi$ on an open subset $G$ of a normed linear space $X$ defined, for $x \in G$ by

$$
\partial \phi(x) \equiv\left\{f \in X^{*} \mid f(y) \leqslant \limsup _{\substack{z \rightarrow x \\ t \rightarrow 0+}} \frac{\phi(z+t y)-\phi(z)}{t} \text { for all } y \in X\right\} .
$$

For any $x \in G, \partial \phi(x)$ is a non-empty weak * .compact convex subset of $X^{*}$ and the mapping $x \rightarrow \partial \phi(x)$ is weak * upper semi-continuous. In Euclidean space, Rademacher's Theorem guarantees that any locally Lipschitz function $\phi$ on an open subset $G$ is differentiable a.e. on $G$. In that case the generalised differential $\partial \phi(x)$ has the particularly useful characterisation as the closed convex hull of the cluster points of $\nabla \phi\left(x_{n}\right)$ for points where $\phi$ is differentiable and where $x_{n}$ converges to $x,[5, p .27$ and p.63]. Given a non-empty closed set $K$ in $X$ generating a distance function $d$, the generalised subdifferential of $d$ is important in characterising tangent and normal cones to the set $K,[5$, p.51].

So for both applications it is useful to determine differentiability properties of distance functions. We are assisted in this study by the fact that distance functions are defined in terms of the norm of the space and in general, distance functions derive their differentiability properties from those for the norm.

A real function $\phi$ on a normed linear space $X$ is said to be Gâteaux differentiable at $x \in X$ if there exists a linear functional $\phi^{\prime}(x)$ on $X$ where, given $\varepsilon>0$ and $\|y\|=1$ there exists a $\delta(\varepsilon, x, y)>0$ such that

$$
\left|\frac{\phi(x+t y)-\phi(x)}{t}-\phi^{\prime}(x)(y)\right|<\epsilon \text { when } 0<|t|<\delta .
$$

The function $\phi$ is said to be Fréchet differentiable at $x$ if there exists a $\delta(\varepsilon, x)>0$ such that the inequality holds for all $\|y\|=1$. The function $\phi$ is said to be uniformly Gâteaux differentiable on a subset $D$ if there exists a $\delta(\varepsilon, y)>0$ such that the inequality holds for all $x \in D$, and is said to be uniformly Fréchet differentiable on a subset $D$ if there exists a $\delta(\varepsilon)>0$ such that the inequality holds for all $x \in D$ and all $\|y\|=1$. When the norm of $X$ is Gâteaux differentiable at $x \neq 0$ we denote the Gâteaux derivative by $f_{x}$ and we note that $\left\|f_{x}\right\|=1$. We say that $X$ has differentiable norm if the norm is differentiable on $\{x:\|x\|=1\}$.

In a normed linear space $X$ with uniformly Gâteaux differentiable norm, Zajicek $[12$, p.300] has shown that the distance function generated by a non-empty closed set 
$K$ always has a significant differentiability property: for each $x \in X \backslash K$ the right hand Gâteaux derivative $d_{+}^{\prime}(x)(y)$ exists and is a concave function in $y$. But also in such a space, the generalised subdifferential $\partial d(x)$ is singleton if and only if $d$ is Gateaux differentiable at $x,[1$, Corollary 9].

In paper [9, Proposition 2] a characterisation of Gâteaux (and Fréchet) differentiability of the distance function was given for a normed linear space with uniformly Gateaux (uniformly Fréchet) differentiable norm. But that characterisation is for the distance function generated by a set where there exists a dense set of points which have best approximating points in the set. The following characterisation does not depend on the existence of such best approximating points in the set.

Proposition 1. Consider a distance function $d$ generated by a non-empty closed set $K$ in a normed linear space $X$ with uniformly Gâteaux (uniformly Fréchet) differentiable norm. For any $x \in X \backslash K, d$ is Gâteaux (Fréchet) differentiable at $x$ if and only if either of the following hold:

(i) $f_{z-u}$ is weak (norm) convergent as $z \rightarrow x,\|z-u\| \rightarrow d(x)$ and $u \in K$;

(ii) $f_{x-v}$ is weak* (norm) convergent as $\|x-v\| \rightarrow d(x)$ and $v \in K$.

Proof: The proof of the Gateaux differentiable characterisation is given in $[\mathbf{1}$, Corollary 9]. We need only consider the Fréchet differentiable characterisation.

(i) Suppose that $f_{z-u}$ is norm convergent as $z \rightarrow x$ and $\|z-u\| \rightarrow d(x)$ where $u \in K$. Then $d$ is Gâteaux differentiable at $x$. Suppose that $d$ is not Fréchet differentiable at $x$. Then there exists an $r>0$ and a sequence $\left\{y_{n}\right\}$ in $X$ where $y_{n} \rightarrow 0$ such that

$$
r\left\|y_{n}\right\|<d\left(x+y_{n}\right)-d(x)-d^{\prime}(x)\left(y_{n}\right)
$$

Choose $z_{n} \in X \backslash K$ such that $\left\|z_{n}-x\right\|<\frac{1}{n}\left\|y_{n}\right\|$ and $u_{n} \in K$ such that

$$
d\left(z_{n}\right)<\left\|z_{n}-u_{n}\right\|<d\left(z_{n}\right)+\frac{1}{n}\left\|y_{n}\right\|
$$

Then

$$
\begin{aligned}
r\left\|y_{n}\right\| & <d\left(z_{n}+y_{n}\right)-d\left(z_{n}\right)-d^{\prime}(x)\left(y_{n}\right)+\frac{2}{n}\left\|y_{n}\right\| \\
& <\left\|z_{n}-u_{n}+y_{n}\right\|-\left\|z_{n}-u_{n}\right\|-d^{\prime}(x)\left(y_{n}\right)+\frac{3}{n}\left\|y_{n}\right\|
\end{aligned}
$$

Since the norm is uniformly Fréchet differentiable, given $0<\varepsilon<r / 2$ there exists a $\delta(\varepsilon)>0$ such that

$$
\left|\left\|z_{n}-u_{n}+y_{n}\right\|-\left\|z_{n}-u_{n}\right\|-f_{z_{n}-u_{n}}\left(y_{n}\right)\right|<\varepsilon\left\|y_{n}\right\| \quad \text { for all }\left\|y_{n}\right\|<\delta
$$


Then

$$
\begin{aligned}
\frac{r}{2}\left\|y_{n}\right\| & <f_{z_{n}-u_{n}}\left(y_{n}\right)-d^{\prime}(x)\left(y_{n}\right)+\frac{3}{n}\left\|y_{n}\right\| \quad \text { for all }\left\|y_{n}\right\|<\delta \\
\frac{r}{2} & <\left\|f_{z_{n}-u_{n}}-d^{\prime}(x)\right\|+\frac{3}{n}
\end{aligned}
$$

and we conclude that $\left\{f_{z_{n}-u_{n}}\right\}$ is not norm convergent to $d^{\prime}(x)$ although $z_{n} \rightarrow x$ and $\left\|z_{n}-u_{n}\right\| \rightarrow d(x)$ for $u_{n} \in K$.

Suppose that $d$ is Fréchet differentiable at $x \in X \backslash K$. Suppose also that there exists a sequence $\left\{z_{n}\right\}$ in $X \backslash K$ where $z_{n} \rightarrow x$ and a sequence $\left\{u_{n}\right\}$ in $K$ where $\left\|z_{n}-u_{n}\right\| \rightarrow d(x)$ but where $\left\{f_{z_{n}-u_{n}}\right\}$ is not norm convergent to $d^{\prime}(x)$. Then there exists an $r>0$ and a subsequence of $\left\{z_{n}-u_{n}\right\}$ such that

$$
\left\|f_{z_{n}-u_{n}}-d^{\prime}(x)\right\|>3 r \text { for all } n
$$

So there exists a sequence $\left\{y_{n}\right\}$ in $X,\left\|y_{n}\right\|=1$ such that

$$
-f_{z_{n}-u_{n}}\left(y_{n}\right)+d^{\prime}(x)\left(y_{n}\right)>3 r \text { for all } n
$$

Since $d$ is Fréchet differentiable at $x$ there exists a $\delta(\varepsilon, x)>0$ such that

$$
\left|d(x+w)-d(x)-d^{\prime}(x)(w)\right|<r\|w\| \text { for all }\|w\| \leqslant \delta
$$

Putting $w_{n} \equiv \delta y_{n}$ we have $\left\|w_{n}\right\|=\delta$ for all $n$, and for each $n$

$$
\begin{aligned}
3 r \delta & <-f_{z_{n}-u_{n}}\left(w_{n}\right)+d^{\prime}(x)\left(w_{n}\right) \\
& <d^{\prime}(x)\left(w_{n}\right)-d\left(x+w_{n}\right)+d(x)+d\left(z_{n}+w_{n}\right)-d\left(z_{n}\right)-f_{z_{n}-u_{n}}\left(w_{n}\right) \\
& +\left\|z_{n}-x\right\|+d\left(z_{n}\right)-d(x) . \\
& <r \delta+\left\|z_{n}-u_{n}+w_{n}\right\|-\left\|z_{n}-u_{n}\right\|-f_{z_{n}-u_{n}}\left(w_{n}\right)+\left\|z_{n}-u_{n}\right\|+\left\|z_{n}-x\right\|-d(x) .
\end{aligned}
$$

Since the norm is uniformly Fréchet differentiable there exists a $0<\delta^{\prime}<\delta$ such that

$$
\left|\left\|z_{n}-u_{n}+w_{n}\right\|-\left\|z_{n}-u_{n}\right\|-f_{z_{n}-u_{n}}\left(w_{n}\right)\right|<r\left\|w_{n}\right\| \text { for all }\left\|w_{n}\right\|<\delta^{\prime}
$$

So

$$
r \delta<\left\|z_{n}-u_{n}\right\|+\left\|z_{n}-x\right\|-d(x) .
$$

But this contradicts $\left\|z_{n}-u_{n}\right\| \rightarrow d(x)$.

(ii) If $d$ is Fréchet differentiable at $x$ then from (i), $f_{x-v}$ is norm convergent as $\|x-v\| \rightarrow d(x)$ and $v \in K$. 
Suppose that $f_{x-v}$ is norm convergent as $\|x-v\| \rightarrow d(x)$ and $v \in K$. Then $d$ is Gâteaux differentiable at $x$. Suppose that $d$ is not Fréchet differentiable at $x$. Then following the corresponding argument in (i) and choosing $v_{n} \in K$ such that

$$
d(x)<\left\|x-v_{n}\right\|<d(x)+\frac{1}{n}\left\|y_{n}\right\|
$$

we find that $f_{x-v_{n}}$ is not norm convergent so $d^{\prime}(x)$ although $\left\|x-v_{n}\right\| \rightarrow d(x)$ and $v_{n} \in K$.

When a distance function does have a dense set of points of Gâteaux differentiability off the set then we have the following characterisation of its generalised subdifferential which is an extension of Clarke's original characterisation in finite dimensional spaces.

COROLLARY 1. In a normed linear space with uniformly Gâteaux differentiable norm, given any non-empty closed set $K$ with distance function $d$ Gâteaux differentiable on a subset $D$ dense in $X \backslash K$, then:

(i) for $x \in X \backslash K, \partial d(x)$ is the weak * closed convex hull of the weak * cluster points of $\left\{d^{\prime}\left(x_{n}\right)\right\}$ for $x_{n} \in D$ and $\left\{x_{n}\right\}$ converging to $x$;

(ii) for $x \in b d y K, \partial d(x)$ is the weak* closed convex hull of 0 and the weak* cluster points of $\left\{d^{\prime}\left(x_{n}\right)\right\}$ for $x_{n} \in D$ and $\left\{x_{n}\right\}$ converging to $x$.

Proof:

(i) From the weak ${ }^{*}$ upper semi-continuity of the subdifferential mapping $x \rightarrow$ $\partial d(x)$ we see that $\partial d(x)$ contains the weak ${ }^{*}$ cluster points of $\left\{d^{\prime}\left(x_{n}\right)\right\}$ for $x_{n} \in D$ and $x_{n} \rightarrow x$. We need only prove the converse containment. Suppose not, then we can strongly separate some $f_{0} \in \partial d(x)$ from the other set which is weak * compact and convex. That is there exists a $y \in X$ and $r>0$ such that

$f_{0}(y)-r>\sup \left\{f(y): f\right.$ is a weak * cluster point of $\left\{d^{\prime}\left(x_{n}\right)\right\}$ for $x_{n} \in D$ and $\left.x_{n} \rightarrow x\right\}$

Since $f_{0} \in \partial d(x)$,

$$
f_{0}(y) \leqslant \limsup _{\substack{z \rightarrow x^{x} \\ t \rightarrow 0+}} \frac{d(z+t y)-d(z)}{t} .
$$

Now there exists $z_{n} \rightarrow x$ and $t_{n} \rightarrow 0+$ such that

$$
f_{0}(y)-\frac{r}{3}<\frac{d\left(z_{n}+t_{n} y\right)-d\left(z_{n}\right)}{t_{n}}
$$

for sufficiently large $n$. Since $x \in X \backslash K, z_{n} \in X \backslash K$ for sufficiently large $n$. Since $D$ is dense in $X \backslash K$, for each $n$ we can choose $x_{n} \in D$ such that $\left\|x_{n}-z_{n}\right\|<\frac{t_{n}^{3}}{3}$. Then

$$
f_{0}(y)-\frac{r}{3}<\frac{d\left(x_{n}+t_{n} y\right)-d\left(x_{n}\right)}{t_{n}}+\frac{2 t_{n}}{3} .
$$


Now by Proposition 1(ii), for each $n$ we can choose $u_{n} \in K$ such that

$$
d^{\prime}\left(x_{n}\right)(y)>f_{x_{n}-u_{n}}(y)-\frac{r}{3} \text { and }\left\|x_{n}-u_{n}\right\|<d\left(x_{n}\right)+\frac{t_{n}^{2}}{3} .
$$

Then

$$
\begin{aligned}
f_{0}(y)-\frac{r}{3} & <\frac{\left\|x_{n}-u_{n}+t_{n} y\right\|-\left\|x_{n}-u_{n}\right\|}{t_{n}}+t_{n} \\
& \leqslant f_{x_{n}-u_{n}+t_{n} y}(y)+t_{n} .
\end{aligned}
$$

Since the norm is uniformly Gateaux differentiable

$$
f_{0}(y)-\frac{r}{3} \leqslant \limsup _{x_{n} \rightarrow x} f_{x_{n}-u_{n}}(y)
$$

So

$$
f_{0}(y)-\frac{2 r}{3} \leqslant \limsup _{x_{n} \rightarrow x} d^{\prime}\left(x_{n}\right)(y) .
$$

But then a weak * cluster point $f_{*}$ of $\left\{d^{\prime}\left(x_{n}\right)\right\}$ as $x_{n} \rightarrow x$ satisfies $f_{0}(y)-\frac{2 r}{3} \leqslant f_{*}(y)$ and this contradicts the separation property.

(ii) follows from [2, Lemma 2].

A characterisation of differentiability of the distance function which depends on the density of points of Gâteaux differentiability off the set also follows immediately from Proposition 1(i).

CoRollakY 2. In a normed linear space $X$ with uniformly Gâteaux (uniformly Fréchet) differentiable norm a non-empty closed set $K$ with distance function $d$ Gâteaux differentiable on a subset $D$ dense in $X \backslash K$ has $d$ Gâteaux (Fréchet) differ-

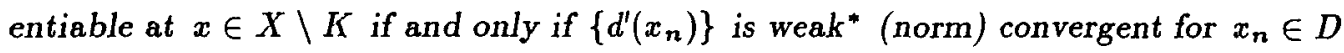
and $\left\{x_{n}\right\}$ converging to $x$.

The following lemma, using a technique of Fitzpatrick [8] exploits the special implications of uniform Gâteaux differentiability of the norm.

LEMMA 1. In a normed linear space $X$ with uniformly Gâteaux (uniformly Fréchet) differentiable norm consider a non-empty closed set $K$ with distance function $d$. If $d$ has the property that there exists a set $P_{+}(K)$ in $X \backslash K$ where for each $x \in P_{+}(K)$ there exists an $\vec{x} \in X,\|\vec{x}\|=1$ such that

$$
\lim _{t \rightarrow 0+} \frac{d(x+t \vec{x})-d(x)}{t}=1
$$


and this limit is approached uniformly for all $x \in P_{+}(K)$, then $d$ is uniformly Gâteaux (uniformly Fréchet) differentiable on $P_{+}(K) \cap B$ where $B$ is a closed ball contained in $X \backslash K$.

Proof: Given that $\varepsilon>0$ and $\|y\|=1$, there exists a $\delta(\varepsilon, y)>0,(\delta(\varepsilon)>0)$ such that

$$
\left|\frac{\|\vec{x}+\lambda y\|-\|\vec{x}\|}{t}-f_{\vec{x}}(y)\right|<\varepsilon \text { for all } x \in P_{+}(K) \text { and } 0<|\lambda| \leqslant \delta,
$$

so

$$
\left|\|\vec{x}-\delta y\|-\|\vec{x}\|+\delta f_{\vec{x}}(y)\right|<\varepsilon \delta .
$$

But also there exists a $\delta^{\prime}(\varepsilon)>0$ such that

$$
\frac{d(x+t \vec{x})-d(x)}{t}>1-\varepsilon \delta \text { for all } x \in P_{+}(K) \text { and all } 0<t<\delta^{\prime} .
$$

Then

$$
\begin{aligned}
d(x+t \delta y)-d(x) & =d(x+t \delta y)-d(x+t \vec{x})+d(x+t \vec{x})-d(x) \\
& >-t(\|\vec{x}-\delta y\|-\|\vec{x}\|-\varepsilon \delta t) \quad \text { for } 0<t<\delta^{\prime} \\
& >t \delta f_{\vec{x}}(y)-2 \varepsilon \delta t .
\end{aligned}
$$

So

$$
\frac{d(x+t y)-d(x)}{t}>f_{\vec{x}^{\prime}}(y)-2 \varepsilon \text { for } 0<t<\delta \delta^{\prime} .
$$

Now since the norm is uniformly Gâteaux differentiable, $d$ has a right-hand derivative $d_{+}^{\prime}(x)$ for all $x \in X \backslash K$ and $-d_{+}^{\prime}(x)(y)$ is sublinear in $y$. But then from (i), for all $x \in P_{+}(K)$,

$$
-d_{+}^{\prime}(x)(y) \leqslant-f_{\vec{x}}(y) \quad \text { for all } y \in X
$$

and so $d$ is Gâteaux differentiable on $P_{+}(K)$ and

$$
d^{\prime}(x)(y)=f_{\vec{x}}(y) \quad \text { for all } y \in K .
$$

Now for any $x \in B$ and $v \in K$ we have $\|x-v\| \geqslant d(B, K)$. Since the norm is uniformly Gâteaux (uniformly Fréchet) differentiable, given $\|y\|=1$ there exists a $\delta^{\prime \prime}(\varepsilon, y)>\left(\delta^{\prime \prime}(\varepsilon)>0\right)$ such that

$$
\left|\frac{\|x-v+t y\|-\|x-v\|}{t}-f_{x-v}(y)\right|<\varepsilon \quad \text { for all } 0<|t|<\delta^{\prime \prime} .
$$


Now for each $x \in P_{+}(K) \cap B$ choose $v \in K$ such that

$$
\|x-v\|<d(x)+\epsilon t \quad \text { where } 0<t<\delta^{\prime \prime} .
$$

Then

$$
\begin{aligned}
d(x+t y)-d(x) & \leqslant\|x+t y-v\|-\|x-v\|+\varepsilon t \\
& <t f_{x-v}(y)+2 \varepsilon t \text { for all } 0<t<\delta^{\prime \prime} .
\end{aligned}
$$

So

$$
\frac{d(x+t y)-d(x)}{t}<f_{x-v}(y)+2 \varepsilon \quad \text { for all } 0<t<\delta^{\prime \prime} .
$$

But this is true for all such choices of $v \in K$. Therefore, since $d$ is Gateaux differentiable at $x$, it follows from Proposition 1.(ii) that

$$
\frac{d(x+t y)-d(x)}{t} \leqslant d^{\prime}(x)(y)+2 \varepsilon \quad \text { for all } 0<t<\delta^{\prime \prime} .
$$

We conclude from (i) and (ii) that $d$ is uniformly Gâteaux (uniformly Fréchet) differentiable on $P_{+}(K) \cap B$.

We should note that the differentiability hypothesis for this lemma is satisfied if $P_{+}(K)$ is a set where there exists an $r>0$ such that for each $x \in P_{+}(K)$ there exists a $z \in X \backslash K$ where $\|x-z\|=r$ and

$$
d(z)=d(x)+\|x-z\|
$$

In the course of the proof of the lemma we have actually established the following result.

COROLlaRY 3. In a normed linear space $X$ with uniformly Gâteaux (uniformly Fréchet) differentiable norm if the distance function $d$ generated by a non-empty closed set $K$ has the property that at some $x \in X \backslash K$, there exists an $\vec{x} \in X,\|\vec{x}\|=1$ where $d_{+}^{\prime}(x)(\vec{x})=1$ then $d$ is Gâteaux (Fréchet) differentiable at $x$.

This corollary should be compared with [1, Theorem 1] for it shows the power of uniform Gâteaux differentiability of the norm with distance functions.

We are now in a position to prove our theorem. This theorem is a generalisation of [9, Theorem] in that assumptions about the existence of best approximation points in the set are replaced by a differentiability assumption on the distance function. The proof is similar to the earlier theorem but is built on Lemma 1 and Corollary 1 established for this more general situation. 
Theonem 1. In a normed linear space $X$ with uniformly Gâteaux (uniformly Fréchet) differentiable norm a non-empty closed set $K$ generates a distance function $d$ which is Gâteaux (Fréchet) differentiable on $X \backslash K$ if there exists a set $P_{+}(K)$ dense in $X \backslash K$ where for each $x \in P_{+}(K)$ there exists an $\vec{x} \in X,\|\vec{x}\|=1$ such that

$$
\lim _{t \rightarrow 0+} \frac{d(x+t \vec{x})-d(x)}{t}=1
$$

and this limit is approached uniformly for all $x \in P_{+}(K)$.

Proof: Consider a closed ball $B$ in $X \backslash K$. It follows from Lemma 1 that $d$ is uniformly Gâteaux (uniformly Fréchet) differentiable on $P_{+}(K) \cap B$. Therefore, given $\varepsilon>0$ and $\|y\|=1$ there exists a $\delta(\varepsilon, y)>0,(\delta(\varepsilon)>0)$ such that

$$
\left|\frac{d(x+t y)-d(x)}{t}-f_{\vec{x}}(y)\right|<\varepsilon \text { for all } x \in P_{+}(K) \cap B \text { and } 0<|t|<\delta .
$$

Then for $x_{1}, x_{2} \in P_{+}(K) \cap B$ and $\|y\|=1$,

$$
\begin{aligned}
\left|\left(f_{\vec{x}_{1}}-f_{\vec{x}_{2}}\right)(y)\right| & \leqslant\left|f_{\vec{x}_{1}}(y)-\frac{d\left(x_{1}+t y\right)-d\left(x_{1}\right)}{t}\right| \\
& +\left|\frac{d\left(x_{1}+t y\right)-d\left(x_{1}\right)}{t}-\frac{d\left(x_{2}+t y\right)-d\left(x_{2}\right)}{t}\right| \\
& +\left|f_{\vec{x}_{2}}(y)-\frac{d\left(x_{2}+t y\right)-d\left(x_{2}\right)}{t}\right| \\
& <2 \varepsilon+\left\|x_{1}-x_{2}\right\| 4 / \delta \text { for all } \delta / 2<|t|<\delta \\
& <6 \varepsilon \text { for all }\left\|x_{1}-x_{2}\right\|<\varepsilon \delta .
\end{aligned}
$$

That is, the mapping $x \rightarrow f_{\vec{x}}(y),\left(x \rightarrow f_{\vec{x}}\right)$ is uniformly continuous on $P_{+}(K) \cap B$. Since $P_{+}(K)$ is dense in $X \backslash K$ this mapping has a unique continuous extension on $B$. But this implies that for any $x \in B$ and sequence $\left\{x_{n}\right\}$ in $P_{+}(K) \cap B$ converging to $x,\left\{f_{\vec{x}_{n}}\right\}$ is weak ${ }^{*}$ convergent, (norm convergent). Since $f_{\vec{x}_{n}}=d^{\prime}\left(x_{n}\right)$ for all $x_{n} \in P_{+}(K)$ it follows from Corollary 2 that $d$ is Gâteaux (Fréchet) differentiable at $x$.

We draw attention to two significant results which follow if we assume that the normed linear space is complete. When we are dealing with a Banach space $X$, a distance function necessarily derives certain differentiability properties from the norm and if the dual $X^{*}$ is rotund then the condition given in Theorem 1 actually implies that the distance function is convex.

Recently, Borwein and Preiss [3] have established a significant extension of Ekeland's Variational Principle. An important application of their result is as follows. 
Proposition 2. In a Banach space with Gâteaux (Fréchet) differentiable norm, every proper lower semi-continuous real function $g$ on an open domain is Gâteaux (Fréchet) subdifferentiable on a dense subset of its domain.

By Gâteaux (Fréchet) subdifferentiability at $x$ we mean that given $\varepsilon>0$ and $\|y\|=1$ there exists a continuous linear functional $f$ on $X$ and a $\delta(\varepsilon, y)>0,(\delta(\varepsilon)>0)$ such that

$$
\frac{g(x+t y)-g(x)}{t} \geqslant f(y)-\varepsilon \text { for all } 0<t<\delta .
$$

The Borwein-Preiss Theorem has the following implication.

Theorem 2. In a Banach space $X$ with uniformly Gâteaux (and Fréchet) differentiable norm, the distance function $d$ generated by a non-empty closed set $K$ is Gâteaux differentiable on a set $D$ dense in $X \backslash K$ ( with $\left\|d^{\prime}(x)\right\|=1$ for all $\left.x \in D\right)$.

Proof: Since $X$ has uniformly Gâteaux differentiable norm we have from Zajicek $[12, \mathrm{p} .300]$ that for each $x \in K \backslash K, d_{+}^{\prime}(x)(y)$ exists for all $y \in X$. But by Proposition $2, d$ is Gâteaux subdifferentiable on a set $D$ dense in $X \backslash K$. So for each $x \in D$ there exists a continuous linear functional $f$ on $X$ such that

$$
d_{+}^{\prime}(x)(y) \geqslant f(y) \text { for all } y \in X
$$

But since $d_{+}^{\prime}(x)(y)$ is concave in $y,[12$, p.300] so $d$ is Gateaux differentiable in $D$. When the norm is also Fréchet differentiable then by Proposition 2 there exists a set $D$ dense in $X \backslash K$ where $d$ is Fréchet subdifferentiable. Then using an argument similar to $\left[1\right.$, Lemma 15] we have that $\left\|d^{\prime}(x)\right\|=1$ for all $x \in D$.

So we deduce from Theorem 2 that in a Banach space with uniformly Gâteaux differentiable norm the generalised subdifferential of a distance function always has such a characterisation as that given in Corollary 2 .

We should point out that the differentiability hypothesis of our theorem is close to the one Vlasov used to deduce the convexity of the set in a Banach space with rotund dual. His result can be stated as follows.

Proposition 3. In a Banach space $X$ with rotund dual $X^{*}$ a non-empty closed set $K$ is convex if its distance function $d$ satisfies

$$
\limsup _{\|y\| \rightarrow 0} \frac{d(x+y)-d(x)}{\|y\|}=1 \text { for all } x \in X \backslash K \text {. }
$$

Now although Theorem 1 applies in a normed linear space which is not necessarily complete, when the normed linear space is complete then a distance function satisfying the hypothesis of Theorem 1 is in fact convex. 
Theorem 3. In a Banach space $X$ with rotund dual $X^{*}$, a non-empty closed set $K$ with distance function $d$ is convex if there exists a set $P_{+}(K)$ dense in $X \backslash K$ with the property that for each $x \in P_{+}\left(K^{-}\right)$there exists an $\vec{x} \in X,\|\vec{x}\|=1$ such that

$$
\lim _{t \rightarrow 0+} \frac{d(x+t \vec{x})-d(x)}{t}=1
$$

and this limit is approached uniformly for all $x \in P_{+}(K)$.

Proof: It is clear that points in $P_{+}(K)$ satisfy Vlasov's differentiability condition. For $x^{\prime} \in(X \backslash K) \backslash P_{+}(K)$ there exists a sequence $\left\{x_{n}\right\}$ in $P_{+}(K)$ converging to $x^{\prime}$. Then given $\varepsilon>0$ there exists a $\delta(\varepsilon)>0$ such that for $x_{n_{0}} \equiv x_{n}+\delta \vec{x}_{n}$,

$$
\begin{aligned}
d\left(x_{n_{0}}\right)-d\left(x^{\prime}\right) & >d\left(x_{n}\right)+(1-\varepsilon)\left\|x_{n_{0}}-x_{n}\right\|-d\left(x^{\prime}\right) \\
& \geqslant(1-\varepsilon)\left\|x_{n_{0}}-x^{\prime}\right\|-(2-\varepsilon)\left\|x_{n}-x^{\prime}\right\|
\end{aligned}
$$

So

$$
\frac{d\left(x_{n_{0}}\right)-d\left(x^{\prime}\right)}{\left\|x_{n_{0}}-x^{\prime}\right\|} \geqslant 1-\varepsilon-\frac{(2-\varepsilon)\left\|x_{n}-x^{\prime}\right\|}{\delta-\left\|x_{n}-x^{\prime}\right\|}
$$

and therefore,

$$
\limsup _{\left\|x_{n_{0}}-x^{\prime}\right\| \rightarrow 0} \frac{d\left(x_{n_{0}}\right)-d\left(x^{\prime}\right)}{\left\|x_{n_{0}}-x^{\prime}\right\|}=1
$$

and we conclude that $K$ is convex by Proposition 3 .

The differentiability property used in Lemma 1 and Theorem 1 for a distance function at a point $x \in X \backslash K$ does not necessarily imply that $x$ has a closest point in $K$ even if $X$ is reflexive. This is shown by Edelstein's example [6, p.6] of a closed set $K$ in an equivalent renorming of $1_{2} \oplus R$ and an open set $U$ in $\left(1_{2} \oplus R\right) \backslash K$ where no point of $U$ has a closest point in $K$. Now Fitzpatrick [7, p.309] has shown that the distance function $d$ in Edelstein's example is Fréchet differentiable in $U$. But then $\left\|d^{\prime}(x)\right\|=1$ for all $x \in U,\left[7\right.$, p.298]. However, since $1_{2} \oplus R$ is reflexive, for each $x \in U$ there exists an $\vec{x},\|\vec{x}\|=1$ such that $d^{\prime}(x)(\vec{x})=1$.

The results of this paper are contributory evidence that it is differentiability properties of the distance function rather than proximinal properties of the set which are important in further development of the two problem areas we indicated in our introduction.

\section{REFERENCES}

[1] J.M. Borwein, S.P. Fitzpatrick and J.R. Giles, 'The differentiability of real functions on normed linear spaces using generalised subgradients', J. Math. Anal. Appl. 128 (1987), 512-534. 
[2] J.M. Borwein and J.R. Giles, 'The proximal normal formula in Banach space', Trans. Amer. Math. Soc. 302 (1987), 371-381.

[3] J.M. Borwein and D. Preiss, 'A smooth variational principle with applications to subdifferentiability and to differentiability of convex functions', Trans. Amer. Math. Soc. 303 (1987), 517-527.

[4] F.H. Clarke, 'Generalised gradients and application', Trans. Amer. Math. Soc. 205 (1975), 247-262.

[5] F.H. Clarke, Optimization and non-smooth analysis Canad. Math. Soc. Series (Wiley, 1983).

[6] Michael Edelstein, 'Weakly proximinal sets', J. Approx. Theory 18 (1976), 1-8.

[7] Simon Fitzpatrick, 'Metric projections and the differentiability of distance functions', Bull. Austral. Math. Soc. 22 (1980), 291-312.

[8] Simon Fitzpatrick, 'Differentiation of real-valued functions and continuity of metric projections', Proc. Amer. Math. Soc. $\theta 1$ (1984), 544-548.

[ө] J.R. Giles, 'Differentiability of distance functions and a proximinal property inducing convexity', Proc. Amer. Math. Soc. 104 no. 2 (1988).

[10] L.P. Vlasov, 'Chebyshev sets and approximatively convex sets', Math. Notes. Acad. Sci. USSR 2 (1967), 600-605.

[11] L.P. Vlasov, 'Almost convex and Chebyshev sets', Math. Nores. Acad. Sci. USSR 8 (1970), 776-779.

[12] L. Zajicek, 'Differentiability of the distance function and points of multivaluedness of the metric projection in Banach space', Czech. Math. J. 33 (1983), 292-308.

Department of Mathematics

The University of Newcastle

New South Wales 2308,

Australia 